\title{
REGIONS OF THE $n$-SPHERE AND RELATED INTEGRALS
}

\author{
by I.D. CURRIE \\ (Received 19th November 1976)
}

\section{Introduction}

In this note the volumes of certain regions in the $n$-sphere will be found in two ways: (a) by using a symmetry argument, (b) by expressing the volumes as repeated integrals over the $(n-1)$-cube. By considering the 4 and 5 spheres and equating the integrals obtained by method (b) to the solution obtained by method (a) we evaluate integrals of the form

$$
I(a, b, c)=\int_{0}^{a} \frac{x \tan ^{-1} x}{\left(b-x^{2}\right) \sqrt{ }\left(c-x^{2}\right)} d x, \quad b>c>0, \sqrt{ } c \geqslant a>0
$$

for certain values of $a, b$ and $c$; it does not appear easy (if indeed it is possible) to evaluate these integrals by direct methods.

These integrals arose in the evaluation of the distribution function of a random variable $W$ defined by Shapiro and Wilk in (1). They define

$$
W=\frac{n\left(\bar{X}-X_{(1)}\right)^{2}}{(n-1) S^{2}}
$$

where $X_{1}, X_{2}, \ldots, X_{n}$ are independent exponential variables, i.e.

and

$$
\begin{aligned}
f_{X}(x) & =e^{-x}, \quad x>0, \\
\bar{X} & =\sum_{i=1}^{n} X_{i} / n, \\
X_{(1)} & =\min \left\{X_{1}, X_{2}, \ldots, X_{n}\right\}
\end{aligned}
$$

$$
S^{2}=\sum_{i=1}^{n}\left(X_{i}-\bar{X}\right)^{2}
$$

The evaluation of the $I(a, b, c)$ was an unexpected bonus.

\section{The Volumes of Two Regions in the $\boldsymbol{n}$-sphere}

Let the volume of the $n$-sphere $\left\{x: \sum_{i=1}^{n} x_{i}^{2} \leqslant 1\right\}$ be denoted by $S_{n}$. Then it is immediately obvious that the volume $U_{n}$ of the region $R_{n}$,

is given by

$$
R_{n}=\left\{x: 0 \leqslant x_{1} \leqslant x_{2} \leqslant \cdots \leqslant x_{n} \quad \text { and } \quad \sum_{i=1}^{n} x_{i}^{2} \leqslant 1\right\}
$$

$$
U_{n}=S_{n} /\left(n ! 2^{n}\right)
$$


The second region $T_{n}$ is defined by

$$
T_{n}=\left\{x: 0 \leqslant \sqrt{ }(1.2) x_{1} \leqslant \sqrt{ }(2.3) x_{2} \leqslant \cdots \leqslant \sqrt{ }(n(n+1)) x_{n} \quad \text { and } \quad \sum_{i=1}^{n} x_{i}^{2} \leqslant 1\right\}
$$

and has volume $V_{n}$ given by

$$
V_{n}=S_{n} /(n+1) !
$$

We prove this as follows: consider the region $R$ in the $(n+1)$-sphere defined by

$$
R=\left\{x: 0 \leqslant \sqrt{ }(1.2) x_{1} \leqslant \sqrt{ }(2.3) x_{2} \leqslant \cdots \leqslant \sqrt{ }(n(n+1)) x_{n} \text { and } \sum_{i=1}^{n+1} x_{i}^{2} \leqslant 1\right\}
$$

and apply the Helmert transformation

$$
\begin{gathered}
x_{i}=\frac{y_{1}+y_{2}+\cdots+y_{i}-i y_{i+1}}{\sqrt{ }(i(i+1))}, \quad i=1, \ldots, n \\
x_{n+1}=\frac{y_{1}+y_{2}+\cdots+y_{n+1}}{\sqrt{ }(n+1)} .
\end{gathered}
$$

Then this transformation maps $R$ into $R^{\prime}$ where

$$
R^{\prime}=\left\{y: y_{1} \geqslant y_{2} \geqslant \cdots \geqslant y_{n+1} \text { and } \sum_{i=1}^{n+1} y_{i}^{2} \leqslant 1\right\} .
$$

By considering the $(n+1)$ ! permutations of the suffices of the $y$ 's we see, by symmetry, that the volume of $R^{\prime}$ is

$$
S_{n+1} /(n+1) \text { ! }
$$

Since the Helmert transformation is orthogonal the volume of $R$ is also given by (1). Then

Suppose the hyperplane $\left\{\boldsymbol{x}: x_{n+1}=a\right\}$ intersects $R$ in a surface $R_{a}$ with area $C_{a}$.

$$
\int_{-1}^{1} C_{a} d a=S_{n+1} /(n+1) !
$$

$R_{a}$ is given by

$$
R_{a}=\left\{x: 0 \leqslant \sqrt{ }(1.2) x_{1} \leqslant \sqrt{ }(2.3) x_{2} \leqslant \cdots \leqslant \sqrt{ }(n(n+1)) x_{n}, x_{n+1}=a, \sum_{i=1}^{n} x_{i}^{2} \leqslant 1-a^{2}\right\} .
$$

and so $R_{0}$ has area $V_{n}$. Now $R_{a}$ is mapped onto $R_{0}$ by the transformation

$$
\begin{gathered}
x_{i}=\sqrt{ }\left(1-a^{2}\right) y_{i}, \quad i=1,2, \ldots, n \\
x_{n+1}=y_{n+1}+a .
\end{gathered}
$$

The Jacobian of this transformation is $\left(1-a^{2}\right)^{n / 2}$ and so

$$
C_{a}=\left(1-a^{2}\right)^{n / 2} V_{n}
$$

Substituting in (2) gives

and the formula for $V_{n}$ follows.

$$
V_{n} \int_{-1}^{1}\left(1-a^{2}\right)^{n / 2} d a=S_{n+1} /(n+1) !
$$




\section{3. $U_{n}$ and $V_{n}$ as Repeated Integrals}

We apply the transformation

$$
x_{i}=\frac{y_{i}}{\sqrt{ }(i(i+1))}, \quad i=1, \ldots, n
$$

to the region $T_{n}$. The Jacobian of this transformation is

$$
1 /(n ! \sqrt{ }(n+1))
$$

and $T_{n}$ transforms into

$$
\left\{y: 0 \leqslant y_{1} \leqslant y_{2} \leqslant \cdots \leqslant y_{n} \text { and } \sum_{i=1}^{n} \frac{y_{i}^{2}}{i(i+1)} \leqslant 1\right\} .
$$

This region is transformed by setting

$$
y_{i}=z_{1} z_{2} \ldots z_{n-i+1}, \quad i=1,2, \ldots, n
$$

The Jacobian of this transformation is $z_{1}^{n-1} z_{2}^{n-2} \ldots z_{n-1}$, and the region is mapped into

$$
\left\{z: 0 \leqslant z_{1}, 0 \leqslant z_{i} \leqslant 1, i=2, \ldots, n \text { and } \sum_{i=1}^{n} \frac{z_{1}^{2} z_{2}^{2} \ldots z_{i}^{2}}{(n-i+1)(n-i+2)} \leqslant 1\right\}
$$

and so we have

$$
V_{n}=\frac{1}{n ! \sqrt{ }(n+1)} \int_{0}^{1} \cdots \int_{0}^{1} \int_{0}^{\theta(z)} z_{1}^{n-1} z_{2}^{n-2} \ldots z_{n-1} d z
$$

where

$$
1 / \theta(z)=\left\{\frac{1}{n(n+1)}+\sum_{i=2}^{n} \frac{z_{2}^{2} z_{3}^{2} \ldots z_{i}^{2}}{(n-i+1)(n-i+2)}\right\}^{1 / 2} .
$$

Carrying out the integration with respect to $z_{1}$ and using the value of $V_{n}$ we find

$$
\begin{aligned}
\int_{0}^{1} \int_{0}^{1} \cdots \int_{0}^{1} \frac{x_{2}^{n-2} x_{3}^{n-3} \ldots x_{n-1}}{\left\{\frac{1}{n(n+1)}+\sum_{i=2}^{n} \frac{x_{2}^{2} x_{3}^{2} \ldots x_{i}^{2}}{(n-i+1)(n-i+2)}\right\}^{n / 2} d x_{2} d x_{3} \ldots d x_{n}} \\
=\frac{n}{\sqrt{ }(n+1)} \cdot \frac{\pi^{n / 2}}{\Gamma(n / 2+1)}, \quad n \geqslant 2 .
\end{aligned}
$$

In order to express $U_{n}$ as a repeated integral we use only the transformation (3) and obtain in the same way

$$
\int_{0}^{1} \int_{0}^{1} \ldots \int_{0}^{1} \frac{x_{2}^{n-2} x_{3}^{n-3} \ldots x_{n-1}}{\left(1+\sum_{i=2}^{n} x_{2}^{2} x_{3}^{2} \ldots x_{i}^{2}\right)^{n / 2}} d x_{2} d x_{3} \ldots d x_{n}=\frac{\pi^{n / 2}}{(n-1) ! 2^{n} \Gamma(n / 2+1)}, n \geqslant 2 .
$$

\section{Applications}

The cases $n=4$ and 5 in expressions (4) and (5) are interesting because if one tries to evaluate the integrals one is led to integrals of the form $I(a, b, c)$ as defined in Section 1. For example, if $n=4$ in (5), and if we integrate out the variables in the 
order $x_{3}$ followed by $x_{2}$, we find that we must evaluate $I(1 / \sqrt{ } 3,3,1)$. The details are elementary and rather tedious but the end result is that

$$
I(1 / \sqrt{ } 3,3,1)=\frac{\sqrt{ } 2}{576} \pi^{2}
$$

By considering the evaluation of (4) and (5) for $n=4$ and 5 , with different orders of integration we can obtain the following results:

\begin{tabular}{|c|c|c|c|c|c|}
\hline Source & $a$ & $b$ & $c$ & $I(a, b, c)$ & Order of integration \\
\hline (5) $n=4$ & $\frac{1}{\sqrt{ }}$ & 3 & 1 & $\frac{\sqrt{2}}{576} \pi^{2}$ & $x_{3}, x_{2}$ \\
(5) $n=4$ & $\frac{1}{\sqrt{3}}$ & 1 & $\frac{1}{2}$ & $\frac{\sqrt{2}}{96} \pi^{2}$ & $x_{3}, x_{4}$ \\
(4) $n=4$ & $\sqrt{\frac{5}{3}}$ & 3 & 2 & $\frac{\pi^{2}}{30}$ & $x_{3}, x_{4}$ \\
(4) $n=4$ & $\frac{1}{\sqrt{3}}$ & 3 & $\frac{1}{2}$ & $\sqrt{ }(10)\left\{\frac{\pi^{2}}{25}-\frac{2 \pi}{15} \tan ^{-1}\left(\sqrt{\frac{5}{3}}\right)\right\}$ & $x_{3}, x_{2}$ \\
(5) $n=5$ & $\frac{1}{\sqrt{ } 3}$ & 3 & 2 & $\frac{\pi^{2}}{20}-\frac{\pi}{6} \tan ^{-1}\left(\sqrt{\frac{5}{3}}\right)$ & $x_{4}, x_{2}, x_{3}$ \\
(4) $n=5$ & $\sqrt{ } 2$ & 3 & 2 & $\frac{\pi^{2}}{12}$ & $x_{4}, x_{2}, x_{3}$ \\
& 1 & 3 & 2 & $\frac{\pi^{2}}{96}$ & - \\
\hline
\end{tabular}

It is easy to show, by using straight forward methods, that

$$
I(\sqrt{ } 2,3,2)=\frac{\pi^{2}}{16}+2 I(1,3,2)
$$

This gives the final entry in the table.

Note: Because of the amount of algebra needed to obtain the above results, all the results were checked by numerical integration.

\section{Comments}

Three obvious questions can be asked:

(i) Can the integrals obtained in 4 be evaluated directly?

(ii) For what other values of $a, b$ and $c$ do nice results like those in 4 hold?

(iii) If the answer to (i) is "no", can other regions in the $n$-sphere be defined which will lead to evaluation of integrals of the form $I(a, b, c)$ ?

Acknowledgement. I acknowledge a very helpful discussion with Professor $\mathbf{H}$. Daniels of Birmingham University. 


\section{REFERENCE}

(1) S. S. SHAPIRO and M. B. WILK, An analysis of variance test for the exponential distribution (complete samples), Technometrics 14 (1972), 355.

DEPaRTMENT OF ACTUARIAL Mathematics \& STATISTICS,

HERIOT-WATT UNIVERSITY,

RICCARTON, CURRIE,

EDINBURGH EH14 4AS 